\title{
Wartezeit für Psychotherapiepatienten - und wie sie zu nutzen ist
}

\author{
$\begin{array}{llll}\text { S. Helbig A. Hähnel } & \text { B. Weigel J. Hoyer }\end{array}$ \\ Institutsambulanz und Tagesklinik für Psychotherapie, Technische Universität Dresden, Deutschland
}

\section{Schlüsselwörter}

Wartezeit · Ambulante Psychotherapie · Gesundheitsversorgung · Selbsthilfe · Prävention, sekundäre

\section{Zusammenfassung}

Wartezeiten von durchschnittlich mehreren Monaten sind auch nach Inkrafttreten des Psychotherapeutengesetzes in der deutschen Psychotherapieversorgung die Regel. Behandlungsbedürftige Störungen, für die ein Behandlungswunsch besteht, unversorgt zu lassen, ist weder unter ethischen, noch praktischen und therapeutischen Gesichtspunkten vertretbar. Aus diesem Grund schlagen viele Praktiker ihren wartenden Patienten niedrigschwellige Selbsthilfeangebote vor, die von psychoedukativen Informationen über Bibliotherapie bis zu Gruppenangeboten reichen. Die vorliegende Arbeit gibt einen Überblick über verschiedene Möglichkeiten, wartende Psychotherapiepatienten gut auf die bevorstehende Therapie vorzubereiten und während der Wartezeit zugleich sekundäre Prävention zu betreiben. Hierbei muss nach unserer Einschätzung vor allem die Maxime gelten, dass die vorgeschlagenen Maßnahmen mit dem Rational der darauf folgenden Therapie vereinbar sein sollten.

\section{Einleitung}

Wartezeiten für Patienten bei der Inanspruchnahme psychotherapeutischer Leistungen sind heute eher die Regel als die Ausnahme. Dabei ist es nicht nur ethisch problematisch, Personen mit hohem Leidensdruck auf Hilfe warten zu lassen, es kann auch direkte negative Konsequenzen für den Therapie-

\begin{tabular}{ll}
\hline KARGER & @ 2004 S. Karger GmbH, Freiburg \\
Fax +497614520714 & Accessible online at: \\
$\begin{array}{l}\text { E-mail Information@Karger.de } \\
\text { www.karger.com }\end{array}$ & www.karger.com/ver
\end{tabular}

Key Words

Waiting time - Outpatient psychotherapy · Health care . Self-help · Prevention, secondary

\section{Summary}

Waiting Time in Psychotherapy - and How to Make Use of $I t$

Even after the new psychotherapy law has been implemented, waiting times of several months remain rather common in the German mental health care system. For ethical, practical, and therapeutic reasons, however, patients who are in serious need of treatment should not be left unattended. Many practitioners therefore suggest self-help treatments such as psychoeducational information, bibliotherapy, or supportive groups to their waiting patients. The present study provides an overview on possibilities of preparing waiting psychotherapy patients for their upcoming therapy as well as implementing secondary prevention during the waiting time. As a basic, we suggest that the proposed methods should be in line with the treatment rationale of the subsequent therapy.

verlauf haben. So zeigen Untersuchungen, dass die Anzahl von Therapieablehnungen mit zunehmender Wartezeit ansteigt [Festinger et al., 1995; Hicks und Hickman, 1994; Loumidis und Shropshire, 1997]. Gleichzeitig belasten unbehandelte psychische Störungen aufgrund der hohen direkten und indirekten Kosten das Gesundheitswesen. So berichten Personen mit aktueller psychischer Diagnose doppelt so viele 
Ausfalltage wie Personen ohne psychische Störung oder Personen mit remittierten psychischen Störungen [Jacobi und Wittchen, 2004]. Der für den Betroffenen entscheidende Aspekt, die subjektive psychosoziale Beeinträchtigung, die aus unbehandelten psychischen Störungen resultiert, ist dabei noch nicht berücksichtigt. Jones und Lodge [1991] sehen in langen Wartezeiten einen Hauptgrund für Unzufriedenheit mit der psychotherapeutischen Versorgungslage sowohl bei Therapeuten als auch bei Patienten. Es ist nicht auszuschließen, dass Wartezeiten die Fehlinanspruchnahme alternativer Behandlungsangebote begünstigen und damit den Krankheitsverlauf komplizieren können. Trotz dieser deutlich defizitären Ausgangslage beschränken sich Verbesserungsvorschläge fast ausschließlich auf Überlegungen zu allenfalls langfristig erreichbaren Veränderungen des Versorgungssystems. Die entstehenden Wartezeiten pragmatisch zu nutzen und somit ihre Nachteile zu minimieren, wurde bisher kaum diskutiert.

\section{Wartezeiten auf Psychotherapie in der Bundesrepublik Deutschland}

Auch nach Inkrafttreten des Psychotherapeutengesetzes (PsychThG) am 1. Januar 1999 entstehen für viele Patienten bei der Inanspruchnahme psychotherapeutischer Leistungen Wartezeiten. Es lassen sich zwei Arten von Wartezeiten unterscheiden: Einmal können Wartezeiten zwischen der Anmeldung des Patienten und dem Erstgespräch bzw. den probatorischen Sitzungen entstehen, zum anderen zwischen der Antragstellung auf Kostenübernahme und dem Therapiebeginn. Häufigkeit und Länge dieser Wartezeiten unterscheiden sich zwischen den Psychotherapeuten, der Art der Richtlinientherapie und der Region (Stadt/Land) [vgl. Fydrich und Kommer, 2004]. Ursachen für diese Wartezeiten können in einem Ungleichgewicht an Inanspruchnahme und Versorgungsangeboten und in den formal-rechtlichen Abläufen bei Psychotherapie gesucht werden. Die Datenlage weist darauf hin, dass sich die Versorgungssituation aufgrund der Einschränkung der therapeutischen Zulassung für approbierte Therapeuten sogar noch verschlechtert hat. Hierfür einige Beispiele.

Zepf et al. [2003] konnten in einer repräsentativen Fragebogenstudie an 1042 Psychotherapeuten in Mecklenburg-Vorpommern, Niedersachsen, Nordrhein-Westfalen und Sachsen zeigen, dass Patienten im Oktober 2000 durchschnittlich 1,9 Monate auf ein diagnostisches Erstgespräch und 4,6 Monate auf einen Behandlungsplatz warten mussten, wohingegen im Zeitraum Juni 1997 bis März 1998 vor Inkrafttreten des PsychThG durchschnittlich 0,7 bzw. 1,8 Monate Wartezeit entstanden [Löcherbach et al., 2000]. Dabei war die durchschnittliche Wartezeit sowohl auf ein Erstgespräch (2,2 Monate) als auch auf einen Therapieplatz (4,8 Monate) bei der Gruppe der Psychologischen Psychotherapeuten verglichen mit Fachärzten für psychotherapeutische Medizin, Psychiatern und Nervenärzten sowie anderen Ärzten mit Zusatzbezeichnun- gen am größten. Einzelaufstellungen der Länder (ohne Mecklenburg-Vorpommern) zeigten darüber hinaus, dass die Wartezeiten auf eine psychologische Psychotherapie in den neuen Bundesländern sogar noch länger sind. Für Sachsen beispielsweise wurde eine durchschnittliche Wartezeit von 2,9 Monaten auf ein Erstgespräch und 6,5 Monaten auf einen Therapieplatz ermittelt [Zepf et al., 2001, 170f].

Der Bund Deutscher Psychologen (BDP) teilte auf Anfrage im Februar 2004 mit, dass die durchschnittliche Wartezeit auf einen Psychotherapieplatz in der Bundesrepublik Deutschland zwischen 3 und 6 Monaten liege, mit Tendenz in Richtung 6 Monaten. In unterversorgten Bereichen wie den neuen Bundesländern, ländlichen Gebieten sowie bei Kindern und Jugendlichen müsse mit Wartezeiten von durchschnittlich 9 Monaten gerechnet werden.

Von den 23 Kassenärztlichen Vereinigungen gaben nur 15 Auskunft über derzeitige durchschnittliche Wartezeiten auf eine Psychotherapie. Dabei kann nur in zwei Regionen, nämlich in Berlin und Bremen, die Versorgungslage als ausreichend eingeschätzt werden. Ausreichend bedeutet, Patienten können dort zu jedem Zeitpunkt einen Therapieplatz erhalten, vorausgesetzt, sie stellen keine Ansprüche an die Art der Behandlung (tiefenpsychologisch, verhaltenstherapeutisch usw.), die behandelnde Person oder die Entfernung des Therapieplatzes zum Wohnort. Damit ist jedoch das Prinzip der freien Therapeutenwahl wesentlich eingeschränkt.

Es ist zu erwarten, dass sich die Wartezeiten auf Erstgespräch und Therapieplatz für Psychotherapiepatienten aufgrund steigenden Bedarfs eher noch verlängern werden [Schulte und Lauterbach, 2002].

\section{Überlegungen zur Nutzung der Wartezeit}

Da nicht zu erwarten ist, dass sich die Versorgungslage in Deutschland in den nächsten Jahren deutlich verbessert, stellt sich die Frage, ob sich unvermeidliche Wartezeiten für den Patienten sinnvoll gestalten lassen. Mögliche Maßnahmen sollten dabei zum einen den Patienten auf Inhalte und Anforderungen der nachfolgenden Therapie vorbereiten und zum anderen auf eine Stabilisierung oder Reduktion von Beschwerden und Symptomen zielen.

Durch Ressourcenaktivierung und Vermittlung störungsspezifischen Wissens sowie allgemein verständliche Informationen über therapeutische Methoden könnten einzelne Therapieinhalte (z.B. Psychoedukation, Korrektur unangemessener Erwartungen, Aktivitätsaufbau) der Therapie vorgelagert und die Behandlungszeit verkürzt werden. Dies wiederum könnte sich positiv auf die Wartezeit nachfolgender Patienten auswirken.

Voraussetzung für die sinnvolle Gestaltung der Wartezeit ist mindestens ein persönlicher Kontakt des Psychotherapeuten mit dem zukünftigen Patienten. Dabei sollte zunächst die Behandlungsindikation abgeklärt werden, um den Patienten bei 
Bedarf an eine andere Versorgungseinrichtung (Krankenhaus, Allgemeinarzt, Beratungsstelle usw.) zu verweisen. Der Kontakt sollte weiterhin genutzt werden, um den Patienten mit für ihn angemessenen Informationen zu versorgen und ihn zur Verwirklichung gegebener Möglichkeiten zu motivieren. Es sollte unbedingt vermieden werden, den Patienten bereits auf das Erstgespräch längere Zeit warten zu lassen, da ansonsten die Gefahr besteht, dass der Patient die Therapie nicht mehr aufnimmt. So fanden Festinger et al. [1995] für Suchterkrankungen (Kokainabhängige), dass über $80 \%$ der Patienten zum Erstgespräch erschienen, wenn es innerhalb von 24 Stunden nach Kontaktaufnahme mit dem Therapeuten stattfand. Lag der Termin für das Erstgespräch 1 Woche später, sank diese Rate auf etwa 20\%. Auch wenn bei diesen Befunden Besonderheiten des Klientels beachtet werden müssen, bleibt anzunehmen, dass lange Wartezeiten einen negativen Einfluss auf die anfängliche Therapiemotivation haben. Auch Westbrook [1995] schlägt vor, diagnostische Sitzungen möglichst bald nach Anmeldung des Patienten anzuberaumen. Zum einen gibt es Hinweise auf Assoziationen eines solchen Vorgehens mit günstigeren Therapieergebnissen, zum anderen empfinden die Patienten solche Kontakte als isolationsreduzierend und unterstützend.

Für die Praxis bedeutet dies, dass Psychotherapeuten wöchentlich feste Termine für Erstgespräche bei Neuanmeldungen reservieren sollten. Besonders effektiv können solche Erstgespräche gestaltet werden, wenn zu diesem Zeitpunkt bereits ein Screening-Fragebogen zu relevanten Problembereichen oder ein anamnestischer Fragebogen vorliegen. Dies setzt jedoch eine persönliche Anmeldung des Patienten voraus, was eher unüblich, da schwierig zu realisieren ist. Alternativ könnten interessierten Patienten solche Fragebögen postalisch zugesandt werden; hier ist jedoch die Kosten-NutzenFrage zu erwägen.

Im Anschluss an das Erstgespräch sollte entschieden werden, ob der Patient weiterverwiesen wird oder auf die Warteliste des jeweiligen Therapeuten kommt. Stellt sich im Erstgespräch beispielsweise heraus, dass der Patient massiv beeinträchtigt oder suizidal ist bzw. eine ambulante Psychotherapie nicht indiziert ist, sollten entsprechende Empfehlungen oder eine Überweisung ausgesprochen werden.

Für dringend behandlungsbedürftige Fälle haben einzelne Kassenärztliche Vereinigungen der alten Bundesländer Datenbanken eingerichtet, an die Psychotherapeuten aktuell verfügbare Therapieplätze melden können. Auf telefonische Anfrage werden diese Informationen an Patienten weitergegeben. Derartige Koordinationsstellen existieren in Bayern, Berlin (Lotsendienst), Hamburg (Psychotherapeutischer Bereitschaftsdienst), Koblenz, Köln und Aachen (ZIP), Rheinhessen, Trier sowie Westfalen-Lippe. Darüber hinaus weist die Kammer für Psychologische Psychotherapeuten und Kinderund Jugendlichenpsychotherapeuten im Land Berlin auf folgende Möglichkeit hin: «Patienten, die unter einer psychischen Störung leiden, können von ihrer Krankenkasse verlan- gen, dass sie die Behandlung durch einen psychologischen Therapeuten bezahlt (Kostenerstattung), der die Erlaubnis zur Ausübung der Heilkunde, aber keine Kassenzulassung besitzt»[Dlubis-Mertens, 2003]. Der Patient muss allerdings nachweisen, dass er bei keinem Vertragspsychotherapeuten innerhalb einer zumutbaren Wartezeit und/oder in angemessener örtlicher Nähe einen Therapieplatz bekommen würde, was für schwere Fälle wohl eher eine unerfüllbare Anforderung darstellt. Zusätzlich wird eine profunde Kenntnis der Versorgungsrechtslage und ein erheblicher Zeitaufwand vorausgesetzt, so dass es zweifelhaft ist, ob dieser Weg für einen behandlungsbedürftigen Patienten mit hohem Leidensdruck gangbar ist.

Sollte eine ambulante Therapie indiziert und die Wartezeit für den Patienten zumutbar sein, können weitere Empfehlungen zur Nutzung der Wartezeit ausgesprochen werden. Im Folgenden werden Möglichkeiten und Maßnahmen zur Überbrückung der Zeit bis zum Therapiebeginn diskutiert.

\section{Psychoedukation}

Zur Vorbereitung der Therapie ist es sinnvoll, dem Patienten Informationen über die Art seiner Störung und deren Zustandekommen zur Verfügung zu stellen. Dies stellt einerseits eine Entlastung und Entpathologisierung für den Patienten dar, der so erfährt, dass er an einer bekannten und behandelbaren Erkrankung leidet. Andererseits helfen diese Informationen, später, in der Therapie ein individuelles Störungsmodell zu erarbeiten, da der Patient seine Symptome einordnen kann und sich eventuell bereits Gedanken über auslösende und aufrechterhaltende Faktoren gemacht hat. Voraussetzung ist die eindeutige Bestimmung der Diagnose des Patienten, d.h. solche Informationen sollten erst nach dem diagnostischen Erstgespräch bzw. der Probatorik eingesetzt werden.

Entstehen Wartezeiten bereits nach Anmeldung des Patienten, können grundlegende Informationen über Psychotherapie und die jeweilige therapeutische Orientierung sowie psychische Erkrankungen allgemein unangemessene Therapieerwartungen des Patienten korrigieren. In seltenen Fällen mag dies beim Patienten zwar zur Wahl eines anderen Therapeuten oder zur Rücknahme des Behandlungswunsches führen; dies scheint in einem frühen Stadium aber sinnvoller als während des Therapieverlaufs. Eine Studie an Studenten konnte nachweisen, dass das Lesen psychoedukativer Materialien zum Thema psychische Störungen die Einstellung zur Inanspruchnahme psychotherapeutischer Angebote verbesserte und Informationen über Psychotherapie mehr positive Erwartungen hinsichtlich einer Therapie induzierten [Gonzales et al., 2002], was für psychoedukative Interventionen spricht.

$\mathrm{Zu}$ diesem Zweck sollte es in jeder Praxis Informationsmaterial (z.B. Broschüren) zu psychotherapeutischen Methoden sowie den häufigsten psychischen Störungen geben, das den Patienten zur Verfügung gestellt werden kann. Ausgewählte 
Tab. 1. Deutschsprachige Links und Internetressourcen*

\begin{tabular}{|c|c|c|}
\hline Problembereich & Internet-Adresse & Anmerkungen \\
\hline Therapeutische Behandlung & www.therapie.de/ & $\begin{array}{l}\text { Im Aufbau begriffenes Nachschlagewerk über Therapieansätze, Hinweise } \\
\text { zur Wahl eines geeigneten Therapeuten }\end{array}$ \\
\hline \multirow[t]{3}{*}{ Psychische Störungen allg. } & www.medizinfo.de/kopfundseele/ & $\begin{array}{l}\text { Sehr informationsreiche Seite zu einer Vielzahl psychischer Störungen mit } \\
\text { kurzen Informationen über Symptome, Stand der Wissenschaft, sehr } \\
\text { allgemeinverständlich }\end{array}$ \\
\hline & www.psychiatrie.de/ & $\begin{array}{l}\text { Mit Download-Archiv für Materialien und Artikel, Möglichkeit zur } \\
\text { Online-Beratung durch Experten }\end{array}$ \\
\hline & www.verhaltenstherapie.at/ & $\begin{array}{l}\text { Patientenratgeber zu den häufigsten Störungsbildern mit Hinweisen auf } \\
\text { Selbsthilfeliteratur }\end{array}$ \\
\hline Angststörungen & www.panik-attacken.de/ & $\begin{array}{l}\text { Informationen über Angststörungen, therapeutische Ansätze, } \\
\text { Literaturempfehlungen, Forum für Betroffene }\end{array}$ \\
\hline Zwang & www.zwaenge.de/ & $\begin{array}{l}\text { Seite der Deutschen Gesellschaft für Zwangserkrankungen; Hinweise für } \\
\text { Betroffene und Therapeuten, umfassende Informationen über } \\
\text { Entstehung und Therapie von Zwängen }\end{array}$ \\
\hline \multirow[t]{2}{*}{ Depression } & www.kompetenznetz-depression.de/ & $\begin{array}{l}\text { Durch BMBF gefördertes Projekt zur Verbesserung der Versorgung und } \\
\text { der Forschung bei Depression; Informationen für Betroffene und } \\
\text { Therapeuten }\end{array}$ \\
\hline & www.depression.de/ & $\begin{array}{l}\text { Kurze Informationen, Selbsttest für Depressionen, Möglichkeit, online } \\
\text { Fragen zu stellen }\end{array}$ \\
\hline Essstörungen & http://ab-server.de/ (Uni Leipzig) & $\begin{array}{l}\text { Linksammlung für weitere Informationen, Forum für Betroffene und } \\
\text { Angehörige, Bewertung von Kliniken }\end{array}$ \\
\hline
\end{tabular}

*Stand: August 2004.

Broschüren findet man unter anderem auf der Homepage des BDP (www.bdp-verband.org/).

Allgemein bietet das Internet sehr gute Möglichkeiten zur Informationsbeschaffung. Hier finden sich neben Seiten von Fachleuten auch Informationen für Betroffene und Angehörige sowie Erfahrungsberichte, Foren, in denen sich Betroffene über ihre Probleme austauschen können, sowie Hinweise und Empfehlungen zur Therapie. Auf einigen Seiten gibt es sogar die Möglichkeit, Experten Fragen zu ausgewählten Problembereichen zu stellen [vgl. Maercker und Lange, 2004]. Allerdings sollten für die Nutzung des Internets immer konkrete Seiten empfohlen werden, da teilweise auch falsche, irreführende oder der Therapiemotivation abträgliche Informationen enthalten sind. Daher ist es nach unserer Auffassung unumgänglich, dass der Therapeut die Seiten, die er empfiehlt, auch selbst überprüft hat. Interessante Internetseiten zu verschiedenen Problembereichen werden in Tabelle 1 zusammengefasst.

\section{Bibliotherapie}

Eine weiterführende Maßnahme zur Therapievorbereitung ist die Bibliotherapie. Darunter werden Methoden zusammengefasst, die in Form schriftlicher Manuale bzw. Tonband- oder Videoaufnahmen Anleitung zur Selbsthilfe bieten. Eine Auswahl derzeit in Deutschland erhältlicher Ratgeber für ausgewählte Problembereiche enthält Tabelle 2.
Obwohl einige bibliotherapeutische Verfahren durchaus den Anspruch erheben, bestimmte Probleme ohne weitere Interventionen behandeln zu können, ist der alleinige Einsatz von Bibliotherapie jedoch problematisch. Zunächst scheinen bibliotherapeutische Maßnahmen nicht für alle Patienten gleich gut geeignet zu sein. Verschiedene Metaanalysen [den Boer, 2004; Gould und Clum, 1993] belegten die besten Effekte von Selbsthilfeansätzen für Ängste und Defizite bei sozialen Kompetenzen. Ebenfalls gut behandelbar waren Depressionen, Kopfschmerzen und Schlaflosigkeit, während Selbsthilfeansätze bei Problemen in Zusammenhang mit Gewohnheiten wie Rauchen oder Trinken eher geringere Erfolge zeigten. Allerdings ist nur ein kleiner Teil der auf dem Markt erhältlichen Programme tatsächlich evaluiert, es bleibt also unklar, ob und wie diese Verfahren wirken. Obwohl einzelne Verfahren empirisch in ihrer Wirksamkeit bestätigt werden konnten [z.B. Gould et al., 1993; Grossman et al., 1991], sind Befunde zu anderen Methoden häufig nicht eindeutig [Febbraro et al., 1999; Hellström und Öst, 1995]. Weitere Probleme bestehen in den hohen Dropout-Raten beim Einsatz solcher Verfahren [Carter et al., 2003; Osgood-Hynes et al., 1998] und in der mangelnden Compliance mit den vorgeschlagenen Interventionen [Broder, 2000; Pantalon und Lubetkin, 1995].

Der Einsatz bibliotherapeutischer Interventionen zur Therapievorbereitung erzielte in ersten Studien dagegen günstige Effekte. So zeigten Treasure et al. [1996], dass bulimische Patienten, die vor der Therapie Selbsthilfemaßnahmen unternahmen, schneller klinisch relevante Verbesserungen erreich- 
Tab. 2. Ausgewählte Selbsthilferatgeber

\begin{tabular}{|c|c|c|c|c|}
\hline Themenbereich & Autor & Titel & Verlag, Jahr & Preis, $€^{*}$ \\
\hline Verhaltenstherapie & Kanfer und Schmelzer & Wegweiser Verhaltenstherapie & Springer Verlag, 2001 & 19,95 \\
\hline \multirow[t]{4}{*}{ Ängste allgemein } & Wolf & Ängste verstehen und überwinden & $\begin{array}{l}\text { PAL Lebenshilfe-Bibliothek, } \\
2001\end{array}$ & 12,80 \\
\hline & Wittchen & Wenn Angst krank macht & Mosaik-Verlag, 1999, ed 2 & 10,70 \\
\hline & $\begin{array}{l}\text { Wittchen, Bullinger-Naber } \\
\text { und Dorfmüller }\end{array}$ & Hexal-Ratgeber Angst & Karger Verlag, 1995 & 5,00 \\
\hline & Schmidt-Traub & Angst bewältigen & Springer Verlag, 2001, ed 2 & 19,95 \\
\hline Soziale Phobie & Fehm und Wittchen & Wenn Schüchternheit krank macht & Hogrefe, 2004 & 12,95 \\
\hline \multirow[t]{2}{*}{ Generalisierte Angststörung } & Wittchen und Schuster & Wenn Angst das Leben lähmt & Mosaik-Verlag, 1998 & 9,95 \\
\hline & $\begin{array}{l}\text { Wittchen, Hoyer, Jacobi } \\
\text { und Schuster }\end{array}$ & $\begin{array}{l}\text { Ihr Therapieratgeber: Generalisierte } \\
\text { Angst }\end{array}$ & Wyeth, 2002 & 10,70 \\
\hline Zwang & Hoffmann & Wenn Zwänge das Leben einengen & PAL Lebenshilfe, 2000 & 12,80 \\
\hline \multirow[t]{4}{*}{ Depression } & Wittchen & Wenn Traurigkeit krank macht & Mosaik-Verlag, 1997 & 10,70 \\
\hline & Wittchen, Möller und Vossen & Hexal-Ratgeber Depression & Karger Verlag, 2002 & 5,00 \\
\hline & Niklewski & Depressionen überwinden & Stiftung Warentest, 2003 & 19,95 \\
\hline & Hell & Welchen Sinn macht Depression? & Rowohlt, 1994 & 8,90 \\
\hline \multirow[t]{2}{*}{ Ess-Störungen } & Gerlinghoff und Backmund & Wege aus der Essstörung & Trias-Verlag, 2004 & 8,00 \\
\hline & $\begin{array}{l}\text { Bundeszentrale für gesund- } \\
\text { heitliche Aufklärung }\end{array}$ & $\begin{array}{l}\text { Ess-Störungen: Bulimie-Magersucht- } \\
\text { Esssucht }\end{array}$ & BzgA, 1999 & gratis \\
\hline
\end{tabular}

*Unverbindliche Preisempfehlung.

ten als solche, die eine Therapie ohne vorangegangene Selbsthilfe begannen. In diesem Sinne empfehlen Baillie und Rapee [2004] für Panikattacken ein gestuftes Vorgehen angefangen bei Psychoedukation und Selbsthilfemaßnahmen bis hin $\mathrm{zu}$ kognitiver Verhaltenstherapie in Abhängigkeit von der Symptomschwere und der allgemeinen Psychopathologie des Patienten.

Selbstverständlich können bibliotherapeutische Materialien auch in die Therapie selbst integriert werden. Hier belegten verschiedene Studien, dass bereits die Kombination von Bibliotherapie mit nur minimalen therapeutischen Kontakten gleichwertige Effekte erzielt [Abbott und Rapee, 2003] bzw. sogar der konventionellen Behandlung [Evans et al., 1999] sowie reiner Selbsthilfe [Carter und Fairburn, 1998] deutlich überlegen ist.

\section{Andere Selbsthilfeansätze}

Andere Möglichkeiten zur Selbsthilfe bieten Selbsthilfegruppen. In den meisten größeren Städten gibt es außerdem Krisentelefone, die häufig rund um die Uhr und anonym in akuten Notsituationen seelischen Beistand leisten und weitere Ansprechpartner benennen können.

Für manche Patienten ist die Teilnahme an einer Selbsthilfegruppe hilfreich. Diese meist privat initiierten Gruppen bieten soziale Kontakte und Unterstützung sowie Informationen über ein bestimmtes Problem und Möglichkeiten des Copings. Gerade für Patienten, die kein ausgeprägtes soziales Netz haben, können derartige Gruppen eine emotionale Stütze und ein Rahmen zum Ausprobieren neuer Verhaltensweisen sein. Trojan [1986] fasst zusammen, dass bessere Krankheitsbewältigung, Aktivitätsaufbau und Kompetenzerweiterung, der Erwerb von Wissen und die Übernahme von Eigenverantwortung positive Wirkungen von Selbsthilfegruppen sind. Informationsmöglichkeiten über regionale und überregionale Selbsthilfegruppen bietet die Nationale Kontakt- und Informationsstelle zur Anregung und Unterstützung von Selbsthilfegruppen (www.nakos.de/). Selbsthilfegruppen sind nicht $\mathrm{zu}$ verwechseln mit professionell angeleiteten Gruppen (z.B. Angehörigenarbeit) und können sich hinsichtlich ihrer Standards und Inhalte stark unterscheiden, was eine Evaluation ihres Nutzens insgesamt sehr erschwert. Es ist auch nicht prinzipiell auszuschließen, dass Selbsthilfegruppen negative Effekte für einzelne Patienten haben können, z.B. durch gegenseitige Stigmatisierung und Schuldzuweisungen. Es empfiehlt sich daher, Hinweise auf sinnvolle Selbsthilfegruppen zu geben, den Patienten aber selbst über seine Teilnahme entscheiden zu lassen. Nützlich für die Praxis ist es, eine Liste mit regionalen Selbsthilfegruppen, Telefonnummern von Krisentelefonen und Adressen von Beratungsstellen ausliegen zu haben. Leider gibt es noch keine umfassenden Dienste für diese Informationen, so dass Adressen und Ansprechpartner vor Ort gesucht werden müssen. 
Für viele Patienten stellt die Anmeldung zur Therapie eine hohe Schwelle dar, die erst einmal überwunden werden muss. Immer noch ist Wissen über Psychotherapie und therapeutische Maßnahmen in der Bevölkerung nur wenig verbreitet und der Gang zum Psychologen ist häufig mit einem sozialen Stigma verbunden. Umso schwieriger und belastender ist es für Patienten, die sich für eine Therapie entschieden haben, Wartezeiten auszuhalten. Wie bereits angesprochen kommt es verständlicherweise bei längeren Wartezeiten auch zu einer erhöhten Rate an Therapieabbrüchen, noch ehe diese überhaupt begonnen haben. Daher ist es wesentlich, Patienten trotz Wartezeit von der Richtigkeit ihrer Entscheidung zu überzeugen und gleichzeitig deren Eigenverantwortung durch Aktivierung zu fördern.

Dies scheint zunächst ein überhöhter Anspruch, denn diese Aufgaben sind selbst im Therapieverlauf unter Anleitung des Therapeuten häufig schwierig zu bewältigen. Es gibt jedoch eine Reihe von Methoden, die schon vor Therapiebeginn helfen, Therapiemotivation aufzubauen und Ressourcen zu aktivieren. Eine auch für die spätere Therapie wertvolle Methode ist die Anleitung des Patienten zur Selbstbeobachtung (selfmonitoring). Besonders effizient kann dies mit strukturierten Tagebüchern geschehen, die für verschiedene Störungsbilder entwickelt wurden [z.B. Marburger Angsttagebuch von Margraf und Schneider, 1990; Marburger Ernährungstagebuch von Tuschen und Florin, 2002]. Dabei lernt der Patient, die verschiedenen Ausprägungsgrade seiner Symptome besser zu differenzieren und kann sich so vorbereitend mit der Symptomregulation beschäftigen. Da in der Wartezeit keine kontinuierliche Kontrolle des Tagebuchführens möglich ist, kann es leicht zu Compliance-Problemen oder falschem Anwenden des Tagebuchs kommen. Es sollten daher folgende Punkte beachtet werden: Jedes Tagebuch sollte zumindest einmal probeweise mit dem Patienten zusammen ausgefüllt werden, damit keine Missverständnisse oder Versagensgefühle beim Patienten entstehen können. Wenn dafür die Zeit fehlt, kann man sich auch mit der Vorlage eines beispielhaft ausgefüllten Bogens behelfen. Allgemein werden kürzere Tagebuchformen besser akzeptiert als längere [Thiele et al., 2002a]. Es sollte darüber hinaus genau festgelegt werden, wann und wie lange das Tagebuch geführt werden sollte. Prinzipiell kommen für die Wartezeit ereigniskontingente und intervallkontingente Tagebücher in Frage. Bei ereigniskontingenten Tagebüchern wird der Patient gebeten, das Auftreten eines bestimmten (problematischen) Verhaltens oder Ereignisses zu protokollieren (z.B. ein Essanfall-Tagebuch), während bei intervallkontingenten Tagebüchern alle Aktivitäten und Ereignisse in einem bestimmten Zeitintervall durch den Patienten dokumentiert werden sollen. Letztere Methode hat den Nachteil, dass sie häufig zeitaufwendig ist und nach einer Weile für den Patienten auch langweilig wird, was ein Absinken der Compliance sowie die Gefahr einer Manipulation des Tagebuchs nach sich zieht [Thiele et al., 2002b]. Der Einsatz intervallkontingenter Tagebücher sollte daher auf 1 bis höchstens 2 Wochen beschränkt werden. In diesem Zeitraum sollte es möglich sein, die relevanten Informationen zu sammeln.

Besonders Patienten mit Unruhezuständen bzw. psychosomatischen Beschwerden können in der Wartezeit vom Erlernen einer Entspannungstechnik profitieren. Hier empfiehlt sich die Progressive Muskelrelaxation nach Jacobson, da diese auch ohne Anleitung gut und schnell erlernbar ist. Verschiedene Krankenkassen bieten zur Unterstützung CDs mit den Übungen und Entspannungsmusik an, die man den Patienten mit nach Hause geben kann.

Eine andere Möglichkeit zur Ressourcenaktivierung kann die Steigerung des allgemeinen Wohlbefindens durch angenehme Aktivitäten darstellen. Hier sollten dem Patienten konkrete Anregungen für mögliche Aktivitäten gegeben werden, die er in seinen Alltag integrieren kann und die im regionalen Umfeld gut realisierbar sind. Die Anwendung solcher Strategien in der Wartezeit ist bislang jedoch noch nicht systematisch untersucht.

Eine allgemeine Variante zur Strukturierung der Wartezeit mit möglichst geringem Aufwand ist die Bereitstellung von Informationsblättern zu Selbsthilfemöglichkeiten in der Wartezeit. Ein solches Informationsblatt wurde an der Institutsambulanz für Psychotherapie der Technischen Universität erarbeitet (Anhang). Ausschlaggebend für die Gestaltung und die Art der gegebenen Informationen war die Frage, welche Wirkmechanismen von Psychotherapie bereits in der Wartezeit einsetzen können. Legt man die von Grawe [1998] postulierten Wirkprinzipien zugrunde, ist neben der Ressourcenaktivierung die Intentionsrealisierung als möglicher Ansatzpunkt zu nennen. Grawe weist insbesondere darauf hin, dass positive Erwartungen im Hinblick auf Veränderungsmöglichkeiten durch die Therapie eine wichtige Moderatorvariable für viele therapeutische Prozesse sein können [Grawe, 1998, $87 \mathrm{ff}]$. Eine positive Erwartung gegenüber der Therapie gekoppelt mit Möglichkeiten, selbst aktiv zu werden, kann beim Patienten zu einer gesteigerten Selbstwirksamkeitserwartung führen. Daher enthält das Informationsblatt nicht nur Anregungen zur gezielten Information über das individuelle Problem des Patienten sowie therapeutische Prinzipien, sondern auch Anregungen zur aktiven Gestaltung der Wartezeit. Dadurch soll der Patient lernen, dass er selbst aktiv zur Steigerung seines Wohlbefindens und damit zu Veränderungsprozessen beitragen kann. Gleichzeitig ist dies der erste Schritt zur Umsetzung der vom Patienten angestrebten Veränderung, die auch Grundlage seines Behandlungswunschs war.

Obwohl es sicher in vielen Institutionen Überlegungen zur Wartezeit gibt, sind der konkrete Einsatz und die Effekte von Interventionen in der Wartezeit bislang kaum dokumentiert. In der Dresdner Ambulanz für Psychotherapie finden derzeit erste Untersuchungen zur Wartezeit statt, unter anderem zur Überprüfung des oben beschriebenen Informationsblatts als Minimalintervention. 
Trotz bislang fehlender empirischer Daten möchten wir Praktiker nachdrücklich ermutigen, vor dem Hintergrund ihrer klinischen Erfahrung den Einsatz und die Möglichkeiten solcher Verfahren zu überdenken. Natürlich können positive Effekte solcher Interventionen nur in klaren Grenzen erwartet werden. Wenn jedoch der Einsatz minimaler Strategien in der Wartezeit auch nur dazu führt, dass der Patient die Therapie mit dem positiven Gefühl beginnt, dass er auch selbst etwas für sich tun kann, ist bereits ein wichtiger Grundstein für den therapeutischen Prozess gelegt.

\section{Kritische Überlegungen}

Obwohl die hier vorgestellten Strategien und Maßnahmen sinnvolle Interventionen zur Überbrückung der Wartezeit darstellen, sollte ihr Einsatz vor Beginn einer Psychotherapie kritisch und gezielt erfolgen. Nicht jede Maßnahme ist für jeden Patienten gleich gut geeignet. Werden die gleichen Methoden bei allen Patienten angewandt, kann das im Einzelfall dazu führen, dass sich Patienten durch Interventionen abgefertigt fühlen, die keinen wirklichen Bezug zu ihrem Problem haben. Es ist weiterhin zu prüfen, ob Patienten die Voraussetzungen für einzelne Maßnahmen erfüllen. Man kann nicht von einem Patienten verlangen, eine Selbsthilfegruppe aufzusuchen, wenn es keine passende Gruppe in der Region gibt oder eine Anreise von $50 \mathrm{~km}$ nötig wäre. Damit verbunden ist auch die Gefahr, Patienten mit Aufgaben zu überfordern. Einem Patienten mit Depressionen oder schwerer Sozialphobie wird es unmöglich sein, zur Ressourcenaktivierung an organisierten Gruppenfahrten in die Umgebung teilzunehmen. Daher ist es unbedingt erforderlich, die in der Wartezeit geplanten Maßnahmen mit dem Patienten abzusprechen und dessen Meinung über die Durchführbarkeit dieser Maßnahmen einzuholen. Bei Misserfolgserlebnissen besteht die Gefahr, dass die Patienten die nachfolgend angebotene Therapie aufgrund geringer Selbstwirksamkeiterwartungen nicht mehr in Anspruch nehmen. In der Studie von Treasure et al. [1996] schlugen immerhin 16\% der Patienten mit Bulimia nervosa, die zuvor bei einer Selbsthilfeintervention keine Verbesserungen erlebt hatten, ein nachfolgendes Therapieangebot aus. Man muss jedoch relativierend sagen, dass die Nichtinanspruchnahme einer einmal angemeldeten Therapie bei konventionellen Wartelisten deutlich höher liegt: So berichten Kenwright und Marks [2003], dass 43\% der angemeldeten $\mathrm{Pa}-$ tienten zumindest die erste Sitzung nicht in Anspruch nahmen, ohne vorher dem Therapeuten abzusagen. Bei Festinger et al. [1995] waren es sogar 58\% der Patienten. Stone und Klein [1999] ermittelten eine allgemeine Absagequote von 20\% für eine angebotene Therapie nach Wartezeit.

Die Akzeptanz von Interventionen in der Wartezeit ist bislang noch wenig untersucht, in einzelnen Studien reicht die Inanspruchnahme freiwilliger Angebote von 15\% (Teilnahme an einer vorbereitenden Selbsthilfegruppe [Stone und Klein,
1999]) bis über 50\% (bibliotherapeutisches Angebot [Whitfield et al., 2001]). Es ist dabei zu beachten, dass die untersuchten Interventionen sehr umfangreich waren. Es ist anzunehmen, dass wenig aufwendige und leicht in den Alltag zu integrierende Maßnahmen stärker akzeptiert werden.

Ein weiteres Problem stellt sich bei Patienten, die negativ auf die gemachten Vorschläge reagieren, die «bereits alles ausprobiert» haben bzw. vergessen, die abgesprochenen Interventionen umzusetzen. Dieses Verhalten weist auf Widerstände oder noch mangelnde Therapiemotivation hin und entspricht Compliance-Problemen, die in der Therapie auftreten. Wenn der Therapeut solche Möglichkeiten vorhalten kann, sind Minimalkontakte, z.B. telefonisch, motivationsfördernd. Ansonsten ist hier der Motivationsaufbau zu Therapiebeginn für den weiteren Therapieverlauf entscheidend.

Ein folgenreiches Problem beim Einsatz von Hinweisen zur Nutzung der Wartezeit entsteht, wenn die Empfehlungen nicht dem entsprechen, was später in der Therapie realisiert werden soll. Beispielsweise können Entspannungsübungen in der Wartezeit einem Angstpatienten signalisieren, dass in der Therapie die Bewältigung und Vermeidung der Angst im Vordergrund stehen. Dementsprechend wird er auf spätere Konfrontationsaufgaben zur Beseitigung der aufrechterhaltenden Mechanismen seiner Ängste nicht vorbereitet sein und skeptisch reagieren. Daher ist es entscheidend, alle angebotenen Interventionen auf die Kompatibilität mit späteren therapeutischen Maßnahmen zu überprüfen sowie dem Patienten zu erläutern, wie sich die Vorschläge in das Gesamtvorgehen der Therapie eingliedern. Zusätzlich empfehlen wir, die im Hinweisblatt angebotenen Vorschläge (siehe Anhang) im Hinblick auf ihre Umsetzbarkeit mit dem Patienten zu besprechen.

Der Einsatz der vorgestellten Maßnahmen impliziert meist einen persönlichen Therapeutenkontakt. Es kann argumentiert werden, dass dies bereits einen hohen Aufwand für den Praktiker darstellt. Wir schlagen daher vor, den Hauptteil unvermeidlicher Wartezeiten erst nach den Abschluss der probatorischen Sitzungen zu legen, sofern dies organisatorisch möglich ist. Damit sind eine Reihe von Vorteilen verbunden, die noch einmal kurz zusammengefasst werden sollen:

1. Der Patient muss nicht lange auf eine Behandlung warten, die für ihn nicht geeignet ist.

2. Ängste und Hemmungen gegenüber der Therapie werden abgebaut; der Patient kann sich bereits mit dem therapeutischen Setting vertraut machen; das Risiko eines vorzeitigen Behandlungsabbruchs sinkt.

3. Adäquate therapievorbereitende und motivierende Strategien zur Nutzung der Wartezeit können geplant werden.

\section{Zusammenfassung}

Wartezeiten in der psychotherapeutischen Versorgung wurden bislang einzig als Kennzeichen der Strukturqualität gewertet. 
Eine effektive Gestaltung dieser Zeit kann jedoch ein wichtiges Merkmal der Prozessqualität von Psychotherapie sein. Der vorliegende Artikel stellt Ansatzmöglichkeiten vor, Wartezeiten für die Therapie nutzbar zu machen. Ziele sind dabei die Therapievorbereitung und die damit verbundene Steigerung ihrer Effizienz sowie die vorübergehende Linderung bzw. Stabilisierung der Beschwerden des Patienten und die Aufrechterhaltung der initialen Therapiemotivation.

Zur Therapievorbereitung empfiehlt sich der Einsatz von psychoedukativen Materialien oder Selbstbeobachtungsaufgaben. Bibliotherapie kann ebenfalls therapievorbereitend oder therapiebegleitend eingesetzt werden, die Tauglichkeit des
Verfahrens für den jeweiligen Patienten und das präsentierte Problem sollte dabei jedoch Berücksichtigung finden. Allgemein können zur Überbrückung der Wartezeit konkrete Hinweise auf Ressourcen mobilisierende Aktivitäten und Hinweise zur Steigerung des Wohlbefindens hilfreich sein. Dabei ist stets zu überprüfen, ob die vorgeschlagenen Maßnahmen mit dem Rational der späteren Therapie logisch und konzeptuell vereinbar sind.

Die meisten der vorgestellten Strategien erfordern minimalen therapeutischen Aufwand; setzen jedoch einen persönlichen Kontakt voraus, in dem Beschwerden und Therapieeignung des Patienten exploriert werden sollten.

\section{Literatur}

Abbott M, Rapee RM: Group treatment and self-help approaches to treating child anxiety and adult social phobia: Does comorbidity affect outcome? Paper presented at the 18th Congress of EABCT, Prag, 2003.

Baillie AJ, Rapee RM: Predicting who benefits from psychoeducation and self help for panic attacks. Behav Res Ther 2004;42:513-527.

Broder MS: Making optimal use of homework to enhance your therapeutic effectiveness. J Rational Emotive Cogn Behav Ther 2000;18:3-18.

Carter JC, Fairburn CG: Cognitive-behavioral selfhelp for binge eating disorder: A controlled effectiveness study. J Consult Clin Psychol 1998;66:616-623.

Carter JC, Olmsted MP, Kaplan AS, McCabe RE, Mills JS, Aimé A: Self-help for bulimia nervosa: A randomised trial. Am J Psychiatry 2003;160:973-978.

Den Boer PCAM, Wiersma D, Van den Bosch RJ: Why is self-help neglected in the treatment of emotional disorders? A meta-analysis. Psychol Med 2004; 34:1-13.

Dlubis-Mertens K: Patienteninfo der Kammer für Psychologische Psychotherapie und Kinder- und Jugendlichenpsychotherapie im Land Berlin. WWW-Dokument 04.06.2004, www.psychotherapeutenkammerberlin.de/fr.htm.

Evans K, Tyrer P, Catalan J, Schmidt U, Davidson K, Dent J, Tata P, Thornton S, Barber JP, Thompson S: Manual-assisted cognitive-behaviour therapy (MACT): A randomized controlled trial of a brief intervention with bibliotherapy in the treatment of recurrent deliberate self harm. Psychol Med 1999;29:19-25.

Febbraro GAM, Clum GA, Roodman A, Wright J: The limits of bibliotherapy: A study of the differential effectiveness of self-administered interventions in individuals with panic attacks. Behav Ther 1999;30: 209-221.

Festinger DS, Lamb RJ, Kountz MR, Kirby KC, Marlowe D: Pretreatment dropout as a function of treatment delay and client variables. Addict Behav 1995; 20:111-115.

Fydrich T, Kommer D: Das Psychotherapeutengesetz: 53 Jahre davor, 5 Jahre danach. Verhaltenstherapie 2004;14:35-41.

Gonzales JM, Tinsley HE, Kreuder KR: Effects of psychoeducational interventions on opinions of mental illness, attitudes towards help seeking, and expectations about psychotherapy in college students. J Coll Student Dev 2002;43:51-63.
Gould RA, Clum GA: A meta-analysis of self-help treatment approaches. Clin Psychol Rev 1993;13: 169-186.

Gould RA, Clum GA, Shapiro DA: The use of bibliotherapy in the treatment of panic: A preliminary investigation. Behav Ther 1993;24:241-252.

Grawe K: Psychologische Psychotherapie. Göttingen, Hogrefe, 1998.

Grossman KS, McNamara JR, Dudley K: Treatment effectiveness of a self-help manual for dating anxiety. J Coll Student Psychother 1991;6:85-106.

Hellström K, Öst L-G: One-session therapists directed exposures vs two forms of manual directed self-exposure in the treatment of spider phobia. Behav Res Ther 1995;33:959-965.

Hicks J, Hickman G: The impact of waiting-list times on client attendance for relationship counselling. Br J Guid Couns 1994;22:175-182.

Jacobi F, Wittchen H-U: «Diagnoseträger» und Patienten: Zur Epidemiologie behandelter und unbehandelter psychischer Störungen in Deutschland. Psychotherapeutenforum 2004;3:17-22.

Jones L, Lodge A: A survey of psychiatric patients: Views of outpatient clinical facilities. Health Bull 1991;49:320-328.

Kenwright M, Marks IM: Improving first attendance for cognitive behaviour therapy by a partial booking appointment method: Two randomised controlled trials. J Ment Health 2003;12:385-392.

Löcherbach P, Henrich T, Kemmer H, Kinstler HJ, Knopp-Vater M, Rieckmann N, Schneider A, Weber I: Indikatoren zur Ermittlung des ambulanten psychotherapeutischen Versorgungsbedarfs, Bd 125, Schriftenreihe des Bundesministeriums für Gesundheit. Baden-Baden, Nomos, 2000.

Loumidis KS, Shropshire JM: Effects of waiting time on appointment attendance with clinical psychologists and length of treatment. Ir J Psychiatry Med 1997; 14:49-54.

Maercker A, Lange A: Psychotherapie im Interent Internet-Therapie. Verhaltenstherapie 2004;14:173. Margraf J, Schneider S: Panik: Angstanfälle und ihre Behandlung. Berlin, Springer, 1990.

Osgood-Hynes DJ, Greist JH, Marks IM, Baer L, Heneman SW, Wenzel KW, Manzo PA, Parkin JR, Spierings CJ, Dottl SL, Vitse HM: Self-administered psychotherapy for depression using a telephone-accessed computer system plus booklet: An open US-UK study. J Clin Psychiatry 1998;59:358-365.
Pantalon MV, Lubetkin BS: Use and effectiveness of self-help books in the practice of cognitive and behavioral therapy. Cog Behav Pract 1995;2:213-228.

Schulte D, Lauterbach W: Neue Therapeuten braucht das Land! Wie groß wird der Bedarf an Psychologischen Psychotherapeuten in der Zukunft sein? Psychother Prax 2002;2:108-110.

Stone WN, Klein EB: The waiting-list group. Int J Group Psychother 1999;49:417-428.

Thiele C, Laireiter AR, Baumann U: Deutschsprachige Tagebuchverfahren in Klinischer Psychologie und Psychotherapie. Z Kl Psych Psychoth 2002a;31: 178-193.

Thiele C, Laireiter AR, Baumann U: Diaries in clinical psychology and psychotherapy: A selective review. Clin Psychol Psychother 2002b;9:1-37.

Treasure J, Schmidt U, Troop N, Todd G: Sequential treatment for bulimia nervosa incorporating a self-care manual. Br J Psychiatry 1996;168:94-98.

Trojan A: Gesundheitsselbsthilfe in Gruppen: Effektivität und Wirkung krankheitsbezogener Selbsthilfegruppen; in Hero S, Ralph B, Christian VF, Ulrich C (eds): Sozialmedizin, Sozialrecht, Gesundheitsökonomie. Berlin, Springer, 1986, pp 137-143.

Tuschen-Caffier B, Florin I: Teufelskreis Bulimie: Ein Manual zur psychologischen Therapie. Münster, Verlag für Psychotherapie, 2002.

Westbrook D: Patient and therapist views of different waiting list procedures. Behav Cog Psychother 1995; 23:169-175.

Whitfield GE, Williams CJ, Shapiro DA: Assessing the take up and acceptability of a self-help room used by patients awaiting their initial outpatient appointment. Behav Cog Psychother 2001;29:333-343.

Zepf S, Mengele U, Marx A: Zur ambulanten psychotherapeutischen Versorgungslage in der Bundesrepublik Deutschland. Gießen, Psychosozial, 2001.

Zepf S, Mengele U, Hartmann S: Zum Stand der ambulanten psychotherapeutischen Versorgung der Erwachsenen in der Bundesrepublik Deutschland. Psychother Psychosom Med Psychol 2003;53:152-162. 
Sehr geehrte Patientin, sehr geehrter Patient,

leider können wir Ihnen aufgrund der Vielzahl von Anmeldungen nicht sofort einen Therapieplatz bei uns anbieten. Zur Überbrückung der Wartezeit und zur Vorbereitung Ihrer psychotherapeutischen Behandlung möchten wir Ihnen folgende Vorschläge unterbreiten:

$>$ Informieren Sie sich über Ihr Krankheitsbild! Für folgende Krankheitsbilder können Sie an unserer Rezeption Informationsblätter und -broschüren erhalten:
$\square$ Panikstörung
$\square$ Soziale Phobie
$\square$ Spezifische Phobien
Zwangsstörungen
$\square$ Depressionen
$\square$ Ess-Störungen
$\square$ Somatoforme Störungen
$\square$ Krankheitsbewältigung

$\square$ Posttraumatische Belastungsstörung

$>$ Nutzen Sie auch das Internet, um sich über Ihr Krankheitsbild und/oder Verhaltenstherapie zu informieren. Wir empfehlen Ihnen u.a. dazu die Seiten: allgemein: $\quad$ http://www.medizinfo.de/kopfundseele/ und http://www.therapie.de/, für Angststörungen: $\quad$ http://www.panikattacken.at/, für Depressionen: $\quad$ http://www.kompetenznetz-depression.de/, für Ess-Störungen: $\quad$ http://www.ab-server.de/, sowie .......

$>$ Achten Sie auf sich selbst! Führen Sie z.B. ein Tagebuch, in dem Sie festhalten, wie sich Ihre Beschwerden (z.B. Angstsymptome, Stimmung oder Schmerzen) im Verlauf eines Tages oder über die Woche hinweg verhalten. Fragen Sie Ihren Therapeuten nach Materialien, die Ihnen dabei helfen können.

$>$ Notieren Sie Anliegen und Fragen, die Sie bezüglich der Therapie haben, sowie Ihre Therapieziele als Vorbereitung auf Ihre erste Therapiesitzung.

Allgemein empfehlen wir Ihnen zur Steigerung Ihres Wohlbefindens:

$>$ Ziehen Sie sich nicht zurück! Rückzug und soziale Isolation fördern die Aufmerksamkeit auf die eigenen Beschwerden und Probleme und erhöhen so den Leidensdruck. Treffen Sie Freunde, unternehmen Sie etwas mit Ihrer Familie oder telefonieren Sie öfter einmal mit einer vertrauten Person.

> Knüpfen Sie neue Kontakte (z.B. über eine psychosoziale Beratungsstelle oder Selbsthilfegruppe, Anmeldung in einem Kurs der Volkshochschule, Besuch eines Fitness-Studios etc.).

$>$ Besuchen Sie eine Bibliothek oder Buchhandlung und schmökern Sie in Publikationen zur Selbsthilfe, die Ihre Problematik betreffen.

$>$ Seien Sie über das Tagesgeschehen um Sie herum informiert und richten Sie damit Ihre Aufmerksamkeit auch einmal von der eigenen Person weg! Lesen Sie regelmäßig eine Tages- oder Wochenzeitung, schauen oder hören Sie Nachrichten.

$>$ Seien Sie im Rahmen Ihrer Möglichkeiten aktiv! Ablenkung und körperliche Betätigung tragen zu Ihrem Wohlbefinden bei. Hier einige Anregungen: Spaziergänge, Wanderungen oder Radfahren, Besuch eines Schwimmbades, Kino, Theater, Konzert, Museum oder Zoo, Stadtbummel, Besuch eines Cafés oder Restaurants, Ausflüge in die nähere Umgebung oder Besichtigung von Sehenswürdigkeiten. Informieren Sie sich auch über Veranstaltungen in Ihrer Nähe bei der Stadtinformation, in Zeitungsbeilagen oder im Internet.

$>$ Entspannen und verwöhnen Sie sich öfters einmal - das ist «Balsam für die Seele»! Kaufen Sie sich eine CD mit Entspannungsübungen oder Musik und nehmen Sie sich täglich Zeit dafür. Versuchen Sie, einmal auszuschlafen oder legen Sie einen „Faulenzertag“ ein. Machen Sie es sich zu Hause mit Ihrer Lieblingsmusik oder bei einem Fernseh-, Video- oder Fotoabend so richtig gemütlich - Probleme und Pflichten sollten Sie dabei «vor der Wohnzimmertür parken». Nehmen Sie ein ausgiebiges Bad oder duschen Sie einmal länger. Vereinbaren Sie einen Frisör- oder Kosmetiktermin. Schlendern Sie durch einen Supermarkt und gönnen Sie sich einmal etwas richtig Leckeres zu essen; vielleicht etwas, was Sie schon immer mal ausprobieren wollten.

Noch ein wichtiger Hinweis zum Schluss: Sollten sich während der Wartezeit wesentliche Veränderungen bei Ihnen ergeben (z.B. drastische Verschlechterung Ihrer Beschwerden, Veränderung des Therapiewunsches, Umzug, Urlaub, Krankenhausaufenthalt, sonstige therapierelevante Fragen oder Informationen), dann rufen Sie uns bitte an: Tel:

Mit herzlichen Grüßen Sprechzeiten:

Ihr Therapeutenteam 